\title{
In-Vitro Inhibitory Effect of Methanol Extracts of Chinese Herbal Drugs on Supercoiling Activity of Bacterial DNA Gyrase
}

\author{
Hui Xu ${ }^{1,2^{*}}$, Huizhi Chen ${ }^{1,2}$, Pan Yao ${ }^{1,2}$, Guodong $\operatorname{Lin}^{3}$, Weiwen Chen ${ }^{1,2}$ \\ ${ }^{1}$ Research Center of Chinese Herbal Resource Science and Engineering, Guangzhou University of Chinese Medicine, \\ Guangzhou, China \\ ${ }^{2}$ Key Laboratory of Chinese Medicine Resource from Lingnan (Guangzhou University of Chinese Medicine), \\ Ministry of Education, Guangzhou, China \\ ${ }^{3}$ School of Chinese Materia Medica, Guangzhou University of Chinese Medicine, Guangzhou, China

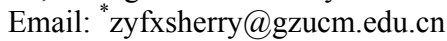

Received December 12, 2012; revised January 18, 2013; accepted January 29, 2013

\begin{abstract}
A large number of Chinese herbal drugs (CHDs) exhibit antibacterial activities both in vivo and in vitro, but until now little is known regarding their inhibitory mechanisms. Bacterial DNA gyrase is a proven target for antibacterial agents. Aim of this study was to investigate the in-vitro inhibitory effect of methanol extracts of CHDs against supercoiling activity of bacterial DNA gyrase. Fifteen CHDs were selected and extracted with methanol, respectively. Inhibitory effect of the extracts on DNA gyrase was tested using gel-based DNA supercoiling assay. Among fifteen CHDs tested, methanol extracts of Lonicerae Japonicae Flos (S2), Taraxaci Herba (S7), Glycyrrhizae Radix et Rhizoma Praeparata cum Melle (S8) demonstrated an obvious inhibitory effect against supercoiling activity of DNA gyrase, and the others were either less active or could not be determined with the present method. Moreover, it was likely that S7 and S8 inhibit gyrase in a concentration-dependent manner. In conclusion, DNA supercoiling assay is a promising method to study the inhibitory activity of CHDs on bacterial DNA gyrase. Some CHDs do have gyrase-inhibitory activity as proposed. Further investigations are needed to elucidate the inhibition mechanism of these CHDs on supercoiling activity of gyrase.
\end{abstract}

Keywords: Chinese Herbal Drugs; Bacterial DNA Gyrase; Supercoiling Activity; Inhibitory Effect

\section{Introduction}

Chinese herbal drugs (CHDs) and formulas have been used for thousands of years in China to treat a wide variety of diseases and nowadays receive more and more recognitions worldwide. Modern researches have demonstrated that a large number of CHDs exhibit antibacterial activities both in vivo and in vitro [1,2]. For example, Lonicerae Japonicae Flos had in vitro obvious antibacterial activity against Staphylococcus aureus, Escherichia coli, Shigella flexneri and Branhamella catarrhlissuch [3]. Galla Chinensis was shown to have potent bacteriostatic effect on methicillin-resistant Staphylococcus aureus (MRSA) as well as vancomycin-resistant Enterococci (VRE) [4,5]. YINHUANG injection, which is prepared of Lonicerae Japonicae Flos and Scutellariae Radix and used for the treatment of tonsillitis, pharyngitis and upper respiratory tract infection in clinic, has been proven to has a good antibacterial effect on Staphylococcus, Pneu-

*Corresponding author. monia and Streptococcus which case respiratory track diseases usually [6]. Since 1980's, scientists have successfully isolated many chemical components, including organic acids, alkaloids, flavonoids, volatile oils, quinonoids and so on, from the CHDs with anti-bacterial activities [7]. But in regard to inhibitory mechanisms, little is known until now, which greatly restricts the antibacterial application of these potential drugs.

Bacterial DNA gyrase, which belongs to type II topoisomerases, is highly conserved and essential for DNA replication and chromosome segregation, as well as for the maintenance of chromosome structure and DNA superhelicity [8]. DNA gyrase is the only known enzyme that negatively supercoils DNA in the presence of ATP [9]. The clinically useful quinolone antibacterial drugs target DNA gyrase by trapping gyrase in an enzymedrug-DNA ternary complex and inhibiting its supercoiling activity, eventually inhibiting the growth of bacteria and killing them $[10,11]$. Other gyrase inhibitors include naturally occuring compounds aminocoumarins, simo- 
cyclinone, cyclothialidine, as well as plasmid-encoded protein $\mathrm{CcdB}$ and microcin B17 [12].

Are there any CHDs that possess anti-bacterial activities targeting bacterial DNA gyrases, either? To present, there is no report on the gyrase inhibition of CHDs. Since CHDs contain tens of thousands of various components, it is very likely that some gyrase inhibitors can be found from them. Hence, we set out to conduct a comprehensive analysis of the inhibitory activity of methanol extracts of CHDs against bacterial DNA gyrase. The results, on one hand, will contribute to understanding of the antibacterial mechanism of CHDs; On the other hand, they may lead to development of novel therapeutic agents.

\section{Materials and Methods}

\subsection{Materials and Reagents}

Fifteen CHDs (Table 1) were bought from Xing-YuanChun Pharmacy, Jingchang Road chain store, Guangzhou. The identification of the CHDs was carried out by Prof. Ruoting Zhan from research center of Chinese herbal resource science and engineering, Guangzhou University of Chinese Medicine, Guangzhou, China. (For simplicity, they were quoted as S1 to S15 in the following text.

\subsection{Preparation of Methanol Extracts of 15 CHDs}

2.0 g powder (through No. 3 sieve) of each CHD was weighed, added with $10 \mathrm{ml}$ methanol and weighed again.
After ultrasonic extraction (power $120 \mathrm{~W}$, frequency 40 $\mathrm{kHz}$ ) for $30 \mathrm{~min}$, the extract was allowed to cool down and weighed again; the loss of weight was replenished with methanol. The extract was filtrated and $2 \mathrm{ml}$ of the successive filtrate was collected and evaporated to nearly dry. The residue was re-dissolved in methanol to obtain the expected concentration.

\subsection{Preparation of Relaxed Plasmid pBR322}

Over $70 \%$ of plasmid pBR322 bought from TAKARA (Dalian, China) was supercoiled. To obtain relaxed DNA, DNA topoisomerase I kit from TAKARA (Dalian, China) was used according to the manufacturer's instruction with some modification. The reaction mixture $(100 \mu \mathrm{l})$ containing $80 \mathrm{ng} / \mu \mathrm{l}$ pBR322, $0.3 \mathrm{U} / \mu \mathrm{l}$ DNA Topoisomerase $\mathrm{I}, 0.3 \%$ bovine serum albumin and $1 \times$ reaction buffer was incubated at $37^{\circ} \mathrm{C}$ for $60 \mathrm{~min}$ and terminated by cooling on ice. Relaxed pBR322 DNA was precipitated with ethanol and dissolved in sterile $\mathrm{H}_{2} \mathrm{O}$.

\subsection{DNA Supercoiling Assay}

Methanol extracts were tested for the inhibitory effect on supercoiling activity of bacterial DNA gyrase by using DNA gyrase kit from TopoGEN (Port Orange, USA) according to the manufacturer's instruction with some modification. For each assay, the mixture $(20 \mu \mathrm{l})$ contained $80 \mu \mathrm{g} / \mu \mathrm{l}$ relaxed pBR322, $0.075 \mathrm{U} / \mu \mathrm{l}$ DNA gyrase, $1 \times$ reaction buffer and $2 \mu \mathrm{l}$ methanol extract of $\mathrm{CHD}$. As

Table 1. List of fifteen CHDs tested in this study.

\begin{tabular}{|c|c|c|c|c|}
\hline No. & Latin Name & Chinese Name (Pinyin) & Family & Nature \\
\hline $\mathrm{S} 1$ & Artemisiae Scopariae Herba & Yinchen & Asteraceae & slightly cold \\
\hline S3 & Zanthoxyli Radix & Liangmianzhen & Rutaceae & neutral \\
\hline $\mathrm{S} 4$ & Andrographis Herba & Chuanxinlian & Acanthaceae & cold \\
\hline $\mathrm{S} 5$ & Phellodendri Chinensis Cortex & Huangbai & Rutaceae & cold \\
\hline S6 & Moutan Cortex & Mudanpi & Ranunculaceae & slightly cold \\
\hline S7 & Taraxaci Herba & Pugongying & Asteraceae & cold \\
\hline $\mathrm{S} 8$ & Glycyrrhizae Radix et Rhizoma Praeparata cum Melle & Zhigancao & Leguminosae & neutral \\
\hline $\mathrm{S} 10$ & Scutellariae Radix & Huangqin & Lamiaceae & cold \\
\hline S11 & Rhei Radix et Rhizoma & Dahuang & Polygonaceae & cold \\
\hline $\mathrm{S} 12$ & Alpiniae Officinarum Rhizoma & Gaoliangjiang & Zingiberaceae & hot \\
\hline $\mathrm{S} 13$ & Isatidis Radix & Banlangen & Cruciferae & cold \\
\hline S14 & Caryophylli Flos & Dingxiang & Myrtaceae & warm \\
\hline S15 & Psoraleae Fructus & Buguzhi & Leguminosae & warm \\
\hline
\end{tabular}


negative control, gyrase was omitted. As positive control, methanol extract was substituted with sterile water. A blank control with $2 \mu \mathrm{l}$ methanol was also included to evaluate the influence of the solvent methanol. The mixtures were incubated at $37^{\circ} \mathrm{C}$ for $60 \mathrm{~min}$ and visualized by agarose gel. Semi-quantification of band intensity was performed using INFINITY-CAPT software Version 14.2 (VILBER LOURMAT, France). The inhibitory effect (IE) of different CHDs was defined as follows:

$$
\mathrm{IE}_{\mathrm{CHD}}=\frac{\text { Intensity }_{\text {supercoiled DNA }}}{\text { Intensity }_{\text {relaxed DNA }}}
$$

\section{Results and Discussions}

By searching literatures and the information database of traditional Chinese Medicine, fifteen potential antibacterial CHDs (S1 to S15) were selected, extracted and tested using DNA supercoiling assay.

The results are shown in Figure 1. At the concentration of $0.4 \mathrm{~g} / \mathrm{ml}$, methanol extracts of S2, S7, and S8 were most effective against supercoiling activity of gyrase, while those of S5 and S10 were less effective, and those of S3, S4, S9, S13 and S15 showed no obvious inhibitory effect on supercoiling activity of gyrase comparing with controls. At the concentration of $0.2 \mathrm{~g} / \mathrm{ml}, \mathrm{S} 1$ was active in DNA supercoiling assay. Impressively, smear or no band could be seen in agarose gel in case of S6, S11, S12 and S14, which suggested that some constituents of these four CHDs may disturb the detection of DNA by agarose gel electrophoresis, and the present method could not be used to access their inhibitory effect against gyrase. The experiments were carried out in duplicate and the results were the same.

Furthermore, we investigated the concentration-effect relationships of S2, S5, S7 and S8. Interestingly, a higher inhibitory activity was observed when a higher concentration extract was added in the reaction mixture (Figure 2). The inhibitory effects of S7 and S8 were positively correlated with the concentration of the extracts between $0.2 \mathrm{~g} / \mathrm{ml}$ and $0.4 \mathrm{~g} / \mathrm{ml}$.

Our results showed that DNA supercoiling assay is a promising method to investigate the inhibitory effect of many CHDs on bacterial DNA gyrase. Some CHDs do inhibit the supercoiling activity of bacterial DNA gyrase in vitro as proposed. Further investigations will be carried out to identify which component/s is/are responsible for the gyrase inhibition of CHDs like S7 and S8. As DNA gyrase is exclusive to the prokaryotic kingdom and essential for the survival of bacteria, it appears to be an ideal target for antibacterial drugs [12]. The finding of novel gyrase inhibitors from CHDs may promote the development of new antibacterial agents and overcome the increasing resistance to available DNA gyrase inhibitors (i.e., quinolone drugs).

As summarized in Table 2, we tried to allocate the gyrase inhibition of CHDs tested with their action according to the theories of Traditional Chinese Medicine [13, 14]. Among five most effective CHDs against gyrase, four of them (S2 and S7, S5 and S10) act to clear away heat. Does this imply the antibacterial agents could probably be found from CHD category that clear heat? Is it likely that heat syndrome of TCM related to bacterial

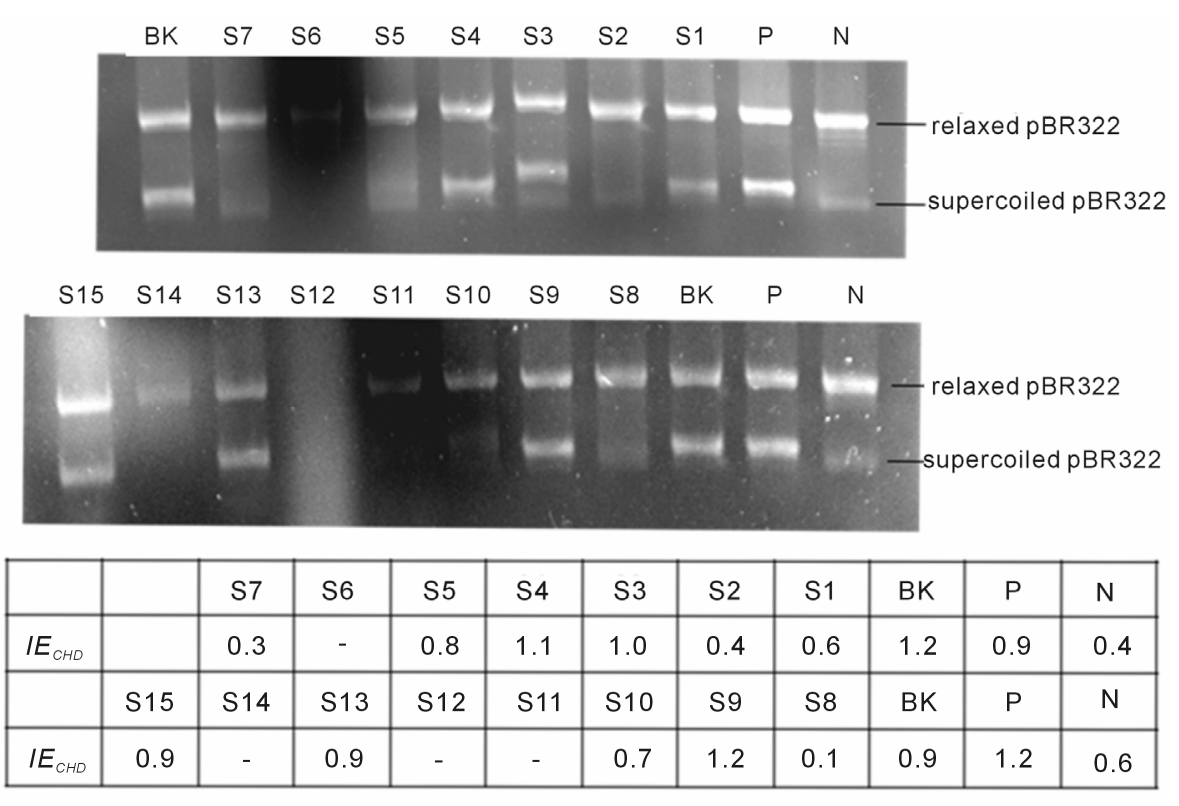

Figure 1. DNA supercoiling assay. (N) negative control without gyrase; (P) positive control with gyrase and sterile $\mathrm{H}_{2} \mathrm{O}$; (BK) blank control with solvent methanol; (S1) reaction with methanol extract of S1 at the concentration of $0.2 \mathrm{~g} / \mathrm{ml}$; (S2-S15) reaction with methanol extract of CHD S2 to $\mathrm{S} 15$ at the concentration of $0.4 \mathrm{~g} / \mathrm{ml}$, respectively. 
Table 2. Actions of fifteen CHDs tested and their in-vitro inhibitory effect on supercoiling activity of bacterial DNA gyrase.

\begin{tabular}{|c|c|c|c|}
\hline No. & Latin Name & Action & Inhibitory Activity* \\
\hline S5 & Phellodendri Chinensis Cortex & \multirow[b]{2}{*}{ To clear away heat and remove dampness } & + \\
\hline S10 & Scutellariae Radix & & + \\
\hline $\mathrm{S} 2$ & Lonicerae Japonicae Flos & \multirow{4}{*}{ To clear away heat and remove toxin } & ++ \\
\hline S4 & Andrographis Herba & & - \\
\hline S7 & Taraxaci Herba & & ++ \\
\hline S13 & Isatidis Radix & & - \\
\hline S6 & Moutan Cortex & To clear away heat and cool the blood & $\mathbf{O}$ \\
\hline S11 & Rhei Radix et Rhizoma & To purge fire and dredge intestines & $\mathbf{O}$ \\
\hline $\mathrm{S} 1$ & Artemisiae Scopariae Herba & To remove damp-heat and relieve jaundice & + \\
\hline S3 & Zanthoxyli Radix & $\begin{array}{l}\text { Antirheumatic drug, to remove wind-dampness } \\
\text { and relieve pain }\end{array}$ & - \\
\hline S8 & Glycyrrhizae Radix et Rhizoma Praeparata cum Melle & \multirow[b]{2}{*}{ Restoratives, to treat asthenic syndrome } & ++ \\
\hline S15 & Psoraleae Fructus & & - \\
\hline S9 & Angelicae Dahuricae Radix & To relieve diaphoretic or exterior Syndrome & - \\
\hline $\mathrm{S} 12$ & Alpiniae Officinarum Rhizoma & \multirow[b]{2}{*}{ To warm up the interior } & $\mathbf{O}$ \\
\hline S14 & Caryophylli Flos & & $\mathbf{O}$ \\
\hline
\end{tabular}

Inhibitory activity ": “++": strong inhibitory effect, $\mathrm{IE}_{\mathrm{CHD}}<0.5$; “+”: week inhibitory effect, $0.5 \leq \mathrm{IE}_{\mathrm{CHD}}<0.9$; “-”: no inhibitory effect, IE $\mathrm{CHD} \geq 0.9$; "O”: not detectable.

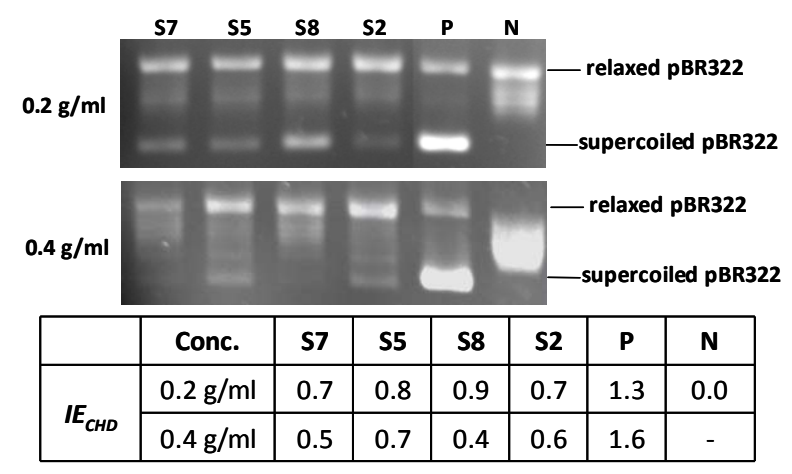

Figure 2. In vitro gyrase inhibition of methanol extracts of $\mathrm{S2}, \mathrm{S5}, \mathrm{S} 7$ and $\mathrm{S8}$ at different concentrations. (N) negative control without gyrase; $(\mathrm{P})$ positive control with gyrase and sterile $\mathrm{H}_{2} \mathrm{O}$; (S2, S5, S7, S8) reaction with methanol extracts of $S 2, S 5, S 7$ and $S 8$, respectively.

infection to some extent? To draw a firm conclusion, more evidence is required. On the other side, S4, the socalled "antibiotic of TCM", as well as S13 are widely used clinically to treat heat syndromes but exhibited little inhibitory activities on bacterial DNA gyrase, which might indicate both of them contain no gyrase inhibitor. In addition, comparison of chemical components of CHDs with gyrase inhibitory activity gave no insight into the relationship between chemical components and the activity.
In summary, in-vitro inhibitory effects of $15 \mathrm{CHDs}$ on bacterial DNA gyrase were studied using DNA supercoiling assay and some interesting results were obtained. Further investigations will help to completely elucidate the inhibition mechanism of these CHDs against gyrase.

\section{Acknowledgements}

This work was sponsored by the Scientific Research Foundation for the Returned Overseas Chinese Scholars, State Administration of TCM, PRC (No. 2005LHR20) and Guangdong Natural Science Foundation, PRC (No. 06301405). We thank Prof. Ruoting Zhan for his kindly help and Dr. Rui He for her critical comments on the manuscript.

\section{REFERENCES}

[1] H. H. Li and L. Ma, "Studies on the Antibacterial Effect of Chinese Medicinal Plants," Journal of Changchun University of Traditional Chinese Medicine, Vol. 26, No. 1, 2010, pp. 136-137.

[2] H. G. Liu, Q. R. Shen and L. M. Liu, "The Development of Traditional Chinese Medicine in Antibacteria," Lishizhen Medicine and Materia Medica Research, Vol. 21, No. 2, 2010, pp. 463-465.

[3] Z. P. Gou and D. G. Wan, "Antibacterial Activity of Varieties of Flos Lonicerae Produced in Sichuan in Vitro by 
Beating Holes Method," Lishizhen Medicine and Materia Medica Research, Vol. 19, No. 3, 2008, pp. 724-725.

[4] Y. B. Tian, Z. H. Chen, M. H. Yang, Y. L. Hu, Q. L. Liu and L. Z. Liu, "In Vitro Bacteriostatic Investigation of 16 Chinese Medical Formula Decoction on MRSA and MSSA," Chinese Journal of Hospital Pharmacy, No. 11, 2009, pp. 897-899.

[5] Y. J. Yang, Y. Q. Liu, J. F. Zhang, X. H. Huang, F. Tan, H. X. Cai, H. P. Huang and Y. Zhu, "In Vitro Antibacterial Activity of Coptidis rhizoma and Galla chinensis on VRE," Journal of Southern Medical University, No. 5, 2008, pp. 819-820.

[6] M. J. Chen, G. Li, L. Gu, J. Liu and S. H. Xiao, "Study on Antibacterial Effect of YINHUANG Injection in Vitro," Lishizhen Medicine and Materia Medica Research, Vol. 18, No. 2, pp. 377-378.

[7] F. Liu and M. R. Kong, "Overview of Antibacterial Activities of Traditional Chinese Medicine," Chinese Journal of Ethnomedicine and Ethnopharmacy, Vol. 2, No. 19, 2010, pp. 14, 38.

[8] J. J. Champoux, "DNA Topoisomerases: Structure, Function, and Mechanism," Annual Review of Biochemistry, Vol. 70, 2001, pp. 369-413.

doi:10.1146/annurev.biochem.70.1.369
[9] R. J. Reece and A. Maxwell, "DNA Gyrase: Structure and Function," Critical Reviews in Biochemistry and Molecular Biology, Vol. 26, No. 3-4, 1991, pp. 335-375. doi:10.3109/10409239109114072

[10] K. Drlica and M. Malik, "Fluoroquinolones: Action and Resistance," Current Topics in Medicinal Chemistry, Vol. 3, No. 3, 2003, pp. 249-282. doi: $10.2174 / 1568026033452537$

[11] A. Maxwell, "DNA Gyrase as a Drug Target," Biochemical Society Transactions, Vol. 27, No. 2, 1999, pp. 4853.

[12] M. Chatterji, S. Unniraman, S. Mahadevan and V. Nagaraja, "Effect of Different Classes of Inhibitors on DNA Gyrase from Mycobacterium smegmatis," Journal of Antimicrobial Chemotherapy, Vol. 48, No. 4, 2001, pp. 479485. doi:10.1093/jac/48.4.479

[13] Chinese Pharmacopoeia Commission, "Pharmacopoeia of the People's Republic of China (Volume I)," People's Medical Publishing House, Beijing, 2010.

[14] T. M. Zhang, "Chinese Medica Materia," Higher Education Press, Beijing, 2008. 